\title{
Uso de ortobiológicos y aloinjerto en el tratamiento de pseudoartrosis con pérdida ósea diafisaria femoral
}

\author{
Use of orthobiologics and allograft in the treatment of \\ pseudoarthrosis with femoral diaphyseal bone loss
}

\begin{abstract}
Martha Brenda Lobato Saucedo, ${ }^{*}$ Rodolfo Sánchez Ayala, ${ }^{\ddagger}$ Miriam Astrit Barriga Magaña, ${ }^{\S}$ Juan Antonio Silva Méndez, ${ }^{\S}$ Israel Gutiérrez-Mendoza, "Álvaro Rodríguez Barrón," Juan Matus Jiménez, ${ }^{* *}$

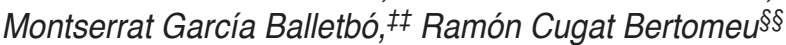

*Médico Residente de $4^{\circ}$ año de la Especialidad de Ortopedia de la UNAM en el Hospital General "Dr. Miguel Silva", Morelia, Michoacán; $¥$ Cirujano Ortopedista de la UNAM. Artroscopia, Ortopedia Regenerativa y Medicina Deportiva en Clínica del Pilar y Hospital Quirón, Barcelona, España. ISAKOS Teaching Center. Módulo Ortopedia Regenerativa FEMECOT. Profesor Titular de Postgrado en la UNAM de la Especialidad de Ortopedia y adscrito al Módulo de Rescate Osteoarticular del Hospital Memorial de Morelia, México; §Cirujano Ortopedista de la UNAM en el Hospital General «Dr. Miguel Silva», Morelia, Michoacán; "Cirujano Ortopedista. Maestría en Ciencias Médicas, Aguascalientes, México; "Maestro en farmacobiología básica adscrito a la Unidad de Investigación del Hospital General "Dr. Miguel Silva», Morelia, Michoacán; **Cirujano Ortopedista de la UNAM en el Hospital General Xoco de la Ciudad de México; ${ }^{\ddagger}$ Especialista en Anatomía Patológica, Máster en Traumatología del Deporte y Doctorado en Medicina por la Universidad de Barcelona, Unidad de Medicina Regenerativa del Hospital Quirónsalud, Fundación García Cugat, Instituto Cugat, Barcelona, España; \$§ Cirujano Ortopedista, Artroscopia, Medicina Deportiva y Doctorado por la Universidad de Barcelona. Mutualidad de Futbolistas, Federación Española de Fútbol-Delegación Cataluña, Fundación García Cugat, Instituto Cugat, Instituto de Traumatología del Hospital Quirónsalud, Barcelona, España.
\end{abstract}

\section{Resumen}

La pseudoartrosis es una complicación a la que se enfrenta el ortopedista, si a ésta se asocia la pérdida ósea, se trata de una cirugía de salvamento. No existe un tratamiento estandarizado en pseudoartrosis con «defecto crítico». Se ha descrito la técnica de membrana inducida, osteogénesis por distracción, entre otras; sin embargo, el tiempo prolongado y el costo que implican han abierto un nuevo panorama en busca de nuevos protocolos. Recientemente se introdujo el "concepto diamante», que no sólo se basa en mejorar la biomecánica, sino que introduce la estimulación biológica como pilar de tratamiento. Se presenta el caso de paciente femenino de 16 años con fractura diafisaria femoral posterior a accidente automovilístico, tratada inicialmente con clavo centromedular que evoluciona a la pseudoartrosis, presentando una pérdida ósea de $5 \mathrm{~cm}$. El tratamiento consistió en retiro de clavo centromedular, resección de segmento óseo no viable, osteosíntesis mediante nuevo clavo centromedular, colocación de aloinjerto diafisario de $7 \mathrm{~cm}$, plasma rico en plaquetas (PRP), aloinjerto de matriz y chips óseos. Logrando consolidación radiográfica y clínicamente restableciendo la función de la extremidad en menor tiempo. Se considera al PRP como el principal ortobiológico en este caso.

Palabras clave: Ortobiológicos, plasma rico en plaquetas, pseudoartrosis, defecto óseo crítico, aloinjerto.

\begin{abstract}
Pseudoarthrosis is a complication that poses a challenge for the orthopedist. If is associated with bone loss, a salvage surgery is indicated. There is no protocol for treatment for pseudoarthrosis with a "critical defect». The induced membrane technique, distraction osteogenesis, among others, has been described; however, the long time and elevated costs involved have opened a new landscape on the research of new protocols. The "diamond concept» which is not only based of improving biomechanics, but also introduces biological stimulation as a pillar of treatment, was recently introduced. We present the case of a 16-year-old female patient with a femoral shaft fracture after a traffic accident, treated initially with a centromedullar blocked nailing that progresses to pseudoarthrosis, presenting a $5 \mathrm{~cm}$ bone loss. Treatment consisted of removing the centromedullar blocked nail, resection of the nonviable bone segment, osteosynthesis with another centromedullar blocked nailing, placement a $7 \mathrm{~cm}$ diaphyseal allograft, platelet-rich plasma (PRP), matrix allograft, and bone chips, thus achieving radiographic and clinical consolidation; restoring limb function in a shorter time, considering the PRP as the main orthobiological element in this case.
\end{abstract}

Keywords: Orthobiologics, platelet rich plasma, pseudoarthrosis, critical bone defect, allograft.

Correspondencia:

Martha Brenda Lobato Saucedo

E-mail: brenda_Is11@outlook.com

Recibido: 12-07-2021. Aceptado: 26-07-2021.
Citar como: Lobato SMB, Sánchez AR, Barriga MMA, Silva MJA, Gutiérrez-Mendoza I, Rodríguez BÁ et al. Uso de ortobiológicos y aloinjerto en el tratamiento de pseudoartrosis con pérdida ósea diafisaria femoral. Orthotips. 2022; 18 (1): 80-85. https://dx.doi.org/10.35366/103737 


\section{Introducción}

La pseudoartrosis se define como la falta de consolidación nueve meses posteriores a la fractura, sin evidencia radiográfica de consolidación durante los tres primeros meses. Weber introdujo un sistema de clasificación basado en la actividad biológica en dos grupos: hipertrófica y atrófica. Si a la pseudoartrosis se añade la pérdida ósea, el cirujano ortopédico se encuentra frente $a$ un verdadero desafío. ${ }^{1-3}$

Respecto a la epidemiología, el retraso de la consolidación en una fractura diafisaria de fémur tratada mediante colocación de clavo centromedular es un problema poco frecuente, existen escasas publicaciones respecto a esta complicación que tiene una gran magnitud en la práctica clínica e importancia en los costos socioeconómicos que conlleva. ${ }^{4}$ Dentro de los factores que predisponen al desarrollo de pseudoartrosis con pérdida ósea podemos enumerar al huésped, comorbilidades y estilo de vida, siendo la inestabilidad el factor mecánico más importante para la no unión. . $^{1,2,5}$

Tamaño «crítico» se refiere al fragmento segmentario que se retira por falta de viabilidad. Algunos autores sugieren que ocurre cuando la longitud del defecto es 2 a 2.5 veces el diámetro del hueso afectado, otros mencionan longitud de defecto superior a $1 \mathrm{~cm}$ y una pérdida superior a $50 \%$ de la circunferencia del hueso. Dentro de los criterios radiográficos para la consolidación, el más importante es la formación de callo óseo, considerando un porcentaje inferior a $5 \%$ de puente óseo como no unión y $25 \%$ de puente óseo consolidación. . $^{6} 9$

Las investigaciones de las propiedades de los diferentes factores de crecimiento contenidos en las plaquetas indican que regulan la proliferación, diferenciación y quimiotaxis celular así como la síntesis de matriz extracelular. El factor de crecimiento transformado beta (TGF- $\beta$ ) estimula el crecimiento, diferenciación y síntesis de matriz extracelular, el factor de crecimiento derivado de plaquetas (PDGF, por sus siglas en inglés) promueve el incremento en la densidad del callo óseo. El PRP es autólogo, no tóxico ni inmunorreactivo, que mediante centrifugación se obtiene una concentración plaquetaria cinco veces mayor que la que se encuentra en la sangre..$^{10-12}$

No existen directrices específicas para el tratamiento óptimo. Frente a esta complicación con pérdida crítica es importante establecer si la extremidad es salvable. Dentro de la literatura se encuentran las siguientes técnicas: inducción de membrana, descrita por Masquelet, la cual consiste en dos fases: la primera consta de la colocación de espaciador de metilmetacrilato en el defecto que busca formar una membrana rica en factores de crecimiento; la segunda fase se completa entre seis y 12 semanas, en la cual se incide cuidadosamente en la membrana, se retira el espaciador y se rellena con injerto óseo autólogo, aloinjerto u otros sustitutos óseos. Osteogénesis por distracción: se trata de formación ósea controlada mediante el uso de fijador externo, descrita en un inicio por llizárov. Injerto de peroné vascularizado, en el cual, las principales desventajas son: morbilidad de la zona donante, requiere especialista microvascular e inestabilidad del tobillo. La más reciente es la bioimpresión tridimensional, siendo un método de fabricación de ingeniería para la construcción de tejidos vivos y órganos análogos, actualmente en investigación. ${ }^{3,6,13}$

El propósito de este reporte de caso es destacar las ventajas del uso de los ortobiológicos y aloinjerto en las pseudoartrosis con defecto crítico, como una gran herramienta para lograr una pronta recuperación tanto clínica como radiográfica.

\section{Presentación del caso}

Paciente femenino de 16 años, estudiante, sin antecedentes de comorbilidades, la cual sufre accidente automovilístico tipo choque frontal, condicionando fractura diafisaria femoral izquierda tratada mediante colocación de clavo centromedular anterógrado de fémur izquierdo, quien evoluciona tórpidamente a la pseudoartrosis aséptica ocho meses posteriores a la intervención. La paciente es valorada en consulta externa, refiriendo dolor a nivel femoral izquierdo, con limitación para la deambulación. A la exploración física se observa miembro pélvico izquierdo con cicatrices quirúrgicas previas, marcha claudicante a expensas del lado afectado con un acortamiento de 2 $\mathrm{cm}$, arcos de movilidad de rodilla ipsilateral limitados con flexión de 100 grados y extensión menor de 5 grados. Cadera izquierda con dolor a la abducción de 45 grados, flexión, extensión y aducción completas. Se valora la radiografía de cirugía previa donde se observan datos de pseudoartrosis atrófica aséptica con clavo centromedular de fémur y alambre (Figura 1).

Se realiza procedimiento quirúrgico, iniciando con la extracción del clavo centromedular de fémur izquierdo, abordando a nivel de la punta del trocánter mayor hacia proximal de $7 \mathrm{~cm}$ para retirar el clavo centromedular previo y otro abordaje lateral para localizar foco de pseudoartrosis. Se retiran tanto alambre incluido en el mismo y $5 \mathrm{~cm}$ de tejido óseo no viable con sierra oscilante, haciendo los cortes de forma perpendicular al eje del fémur, se perfora con broca $3.2 \mathrm{~mm}$ en los 
bordes de la cortical viable, haciendo varios orificios sangrantes tanto en la cortical del segmento proximal y distal, se realiza rimado del canal medular. De igual forma se prepara el aloinjerto, segmento diafisario de 12 cm Biograft, irrigando con solución fisiológica, se hacen los cortes de éste con sierra oscilante para dejarlo de 7 $\mathrm{cm}$, ya con el fragmento de aloinjerto a utilizar se coloca clavo centromedular anterógrado de fémur Synthes 11 $\times 380 \mathrm{~mm}$ bajo técnica AO. Se introduce el clavo centromedular de fémur a través del segmento diafisario a utilizar logrando una reducción a 100\% y la simetría de ambos miembros pélvicos. Por último, se prepara el plasma rico en factores de crecimiento (PRGF, por sus siglas en inglés), técnica BTI-Endoret, se toma la fracción 1 y 2 activada con cloruro de calcio a $10 \%$,

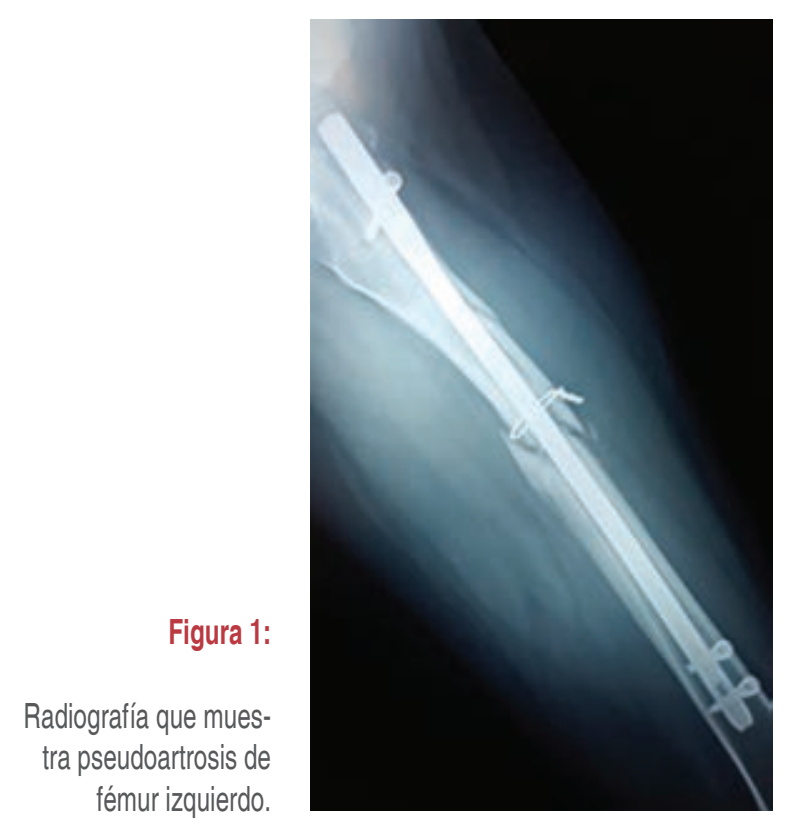

ésta se mezcla con chips óseos y al pasar 10 minutos se obtiene la formulación tipo 3 del PRGF, es decir, la fracción 2 activada y coagulada. Se colocan los chips con el PRGF en los sitios de unión tanto proximal y distal del aloinjerto diafisario con el fémur de la paciente, adicionalmente se coloca matriz ósea desmineralizada en los mismos sitios de unión mencionados (Figura 2).

El seguimiento clínico y radiográfico se realizó a los tres, ocho, 12, 20 y 48 meses con resultados clínicos y radiográficos favorables, correlacionados con la funcionalidad y recuperación de la dismetría de la extremidad afectada (Figuras 3 y 4). La rehabilitación temprana fue fundamental, ya que desde el segundo día de postoperada se inició con ejercicios isométricos, además de promover la flexión y extensión completa de la rodilla del miembro pélvico afectado. Después del retiro de puntos a las dos semanas de la cirugía se inicia descarga parcial progresiva con muletas y durante el segundo mes de postoperada se inicia la deambulación de forma independiente. Se observó a los tres meses formación de puentes óseos, una franca integración del aloinjerto a los 12 meses y con un seguimiento satisfactorio a los cuatro años (Figura 5).

\section{Discusión}

En nuestro caso, la paciente con una pérdida ósea de $7 \mathrm{~cm}$ en fémur, con la técnica descrita inicialmente en un solo tiempo quirúrgico logró arcos de movilidad completos, fuerza muscular normal, sin dolor, deambulación de forma independiente e integración en su totalidad de sus actividades a los tres meses de postoperada, con una franca integración del injerto desde los 12 meses hasta lograr consolidación completa a los 20 meses. Cabe destacar que hubo una pérdida de seguimiento de los 12 a los 20 meses de postoperada, donde no
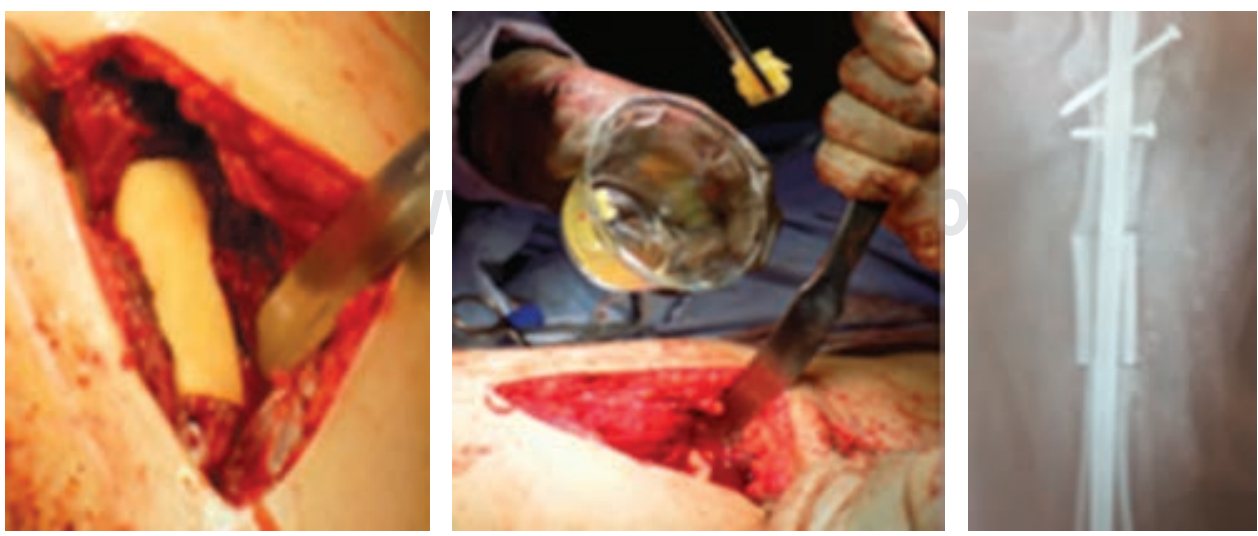

Figura 2:

Radiografía postoperatoria, colocación de aloinjerto, plasma rico en plaquetas, chips y matriz ósea. 
Figura 3:

A) Muestra control tres meses del postquirúrgico,

B) 12 meses y

C) 20 meses.
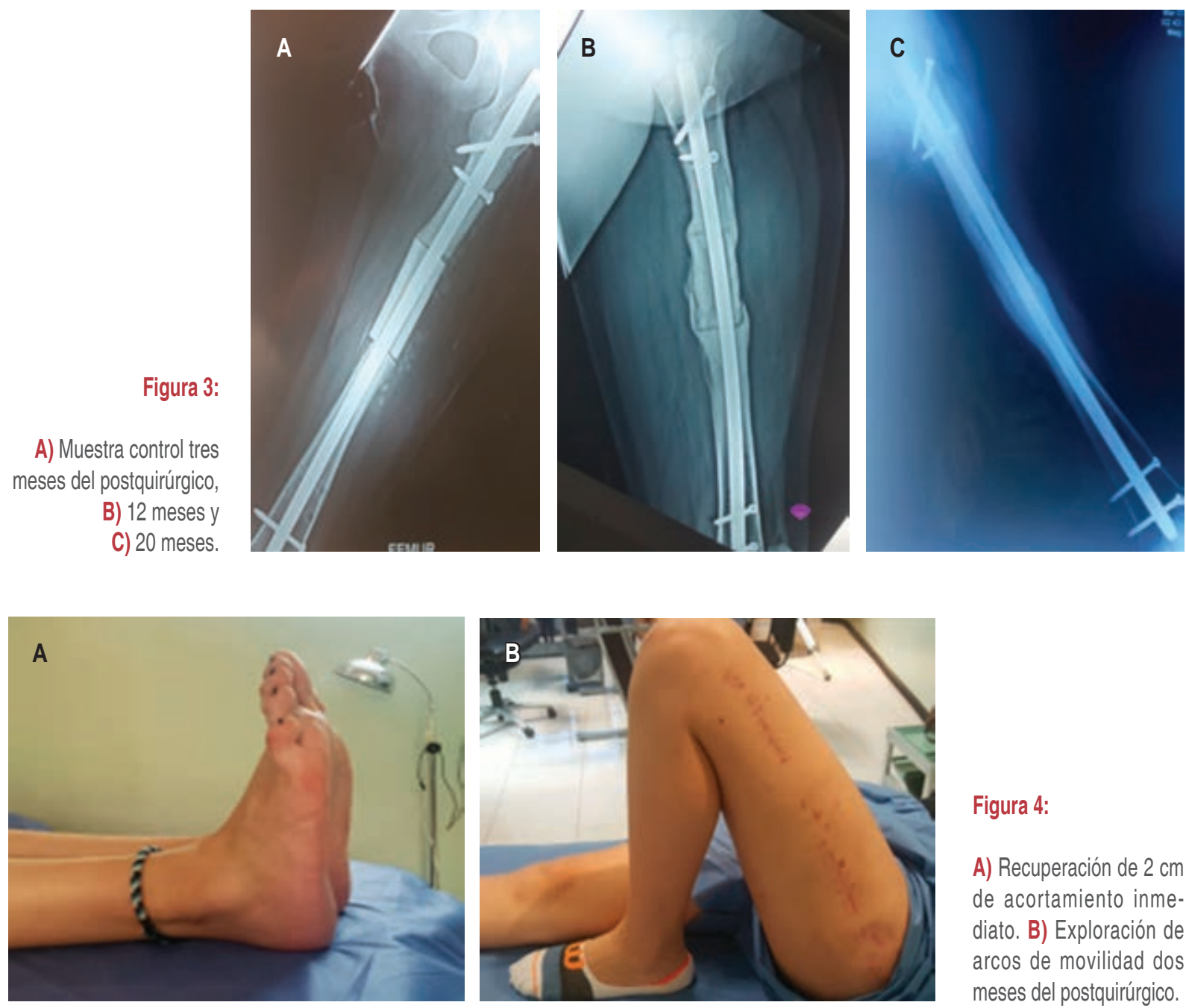

Figura 4:

A) Recuperación de $2 \mathrm{~cm}$ de acortamiento inmediato. B) Exploración de arcos de movilidad dos meses del postquirúrgico.

se pudo observar la evolución radiográfica durante esos ocho meses. Hoy en día a 48 meses de la cirugía nuestra paciente conserva la consolidación completa, clínicamente asintomática y con arcos de movilidad completos, integrada a sus actividades de manera normal.

En este reporte se revisó la literatura nacional e internacional respecto a las diversas técnicas de tratamiento en la pseudoartrosis. La técnica de membrana inducida de Masquelet descrita desde 1980 consta de dos fases, la formación de la membrana es la clave de su éxito. La membrana está compuesta por colágeno tipo I y III, proteína morfogenética ósea 2 (BMP2, por sus siglas en inglés), TGF- $\beta$ (factor de crecimiento transformante $\beta$ ), factores proangiogénicos (VEGF, ANGII y FGF2), IL-6, TNF- $\alpha$, IL-8, IL-10, células supresoras derivadas de los mieloides (MDSC, por sus siglas en inglés) se planteó la hipótesis de que células endoteliales vasculares y osteoblastos favorecen la angiogénesis. En ausencia de membrana injertos óseos de más de 4-6 cm se reabsorben; en comparación con lo observado en nuestro caso, el aloinjerto de $7 \mathrm{~cm}$ preserva su integridad a cuatro años de seguimiento. En un estudio realizado por el Dr. Masquelet un total de 35 pacientes lograron la unión injerto-hueso en un rango de seis a 17 meses. El metaanálisis de Morelli muestra un tiempo de unión que oscila entre seis y 211 semanas. Se ha observado una tasa de éxito de aproximadamente $80-82 \%$ de las técnicas realizadas. Los estudios de Masquelet y de Morelli coinciden con nuestro caso en cuanto al rango de tiempo de consolidación; sin embargo, la ventaja de nuestra técnica es que consta de un solo tiempo quirúrgico con una integración del injerto desde los 12 meses. En defectos grandes mediante técnica de os- 
teogénesis por distracción se ha reportado un tiempo de 18 meses para valorar resultados, teniendo como desventaja sólo el uso de fijador externo a diferencia de nuestro caso, donde se utiliza clavo centromedular, con lo cual nos permite el apoyo temprano de la extremidad y una rehabilitación temprana. La falta de un estudio prospectivo comparativo con otros métodos de tratamiento en pseudoartrosis es evidente. ${ }^{14,15}$

En la actualidad el enfoque en el tratamiento pone como piedra angular la base biológica. El plasma rico en plaquetas (PRP) es autólogo, no tóxico, ni inmunorreactivo, contiene una cuenta plaquetaria cinco veces mayor que la sangre normal. ${ }^{10-12}$ EI PRP se caracteriza por una gran composición de factores de crecimiento, dentro de los más importantes, similar a la insulina-1 (IGF-1) que es un mediador en el crecimiento y reparación musculoesqueléticos. ${ }^{16}$

La investigación del PRP para regeneración ósea se centra en el aprovechamiento de la cascada de los factores de crecimiento en el hematoma nativo fracturario. EI PRP contiene varios factores de crecimiento con propiedades mitogénicas y quimiotácticas, los cuales incluyen los factores de crecimiento derivado de las plaquetas (PDFG, por sus siglas en inglés), el factor de crecimiento insulínico (IGF, por sus siglas en inglés), factor de crecimiento fibroblástico (FGF, por sus siglas en inglés), factor de crecimiento transformado beta (TGF- $\beta$, por sus siglas en inglés) y el factor de crecimiento vascular endotelial (VEGF, por
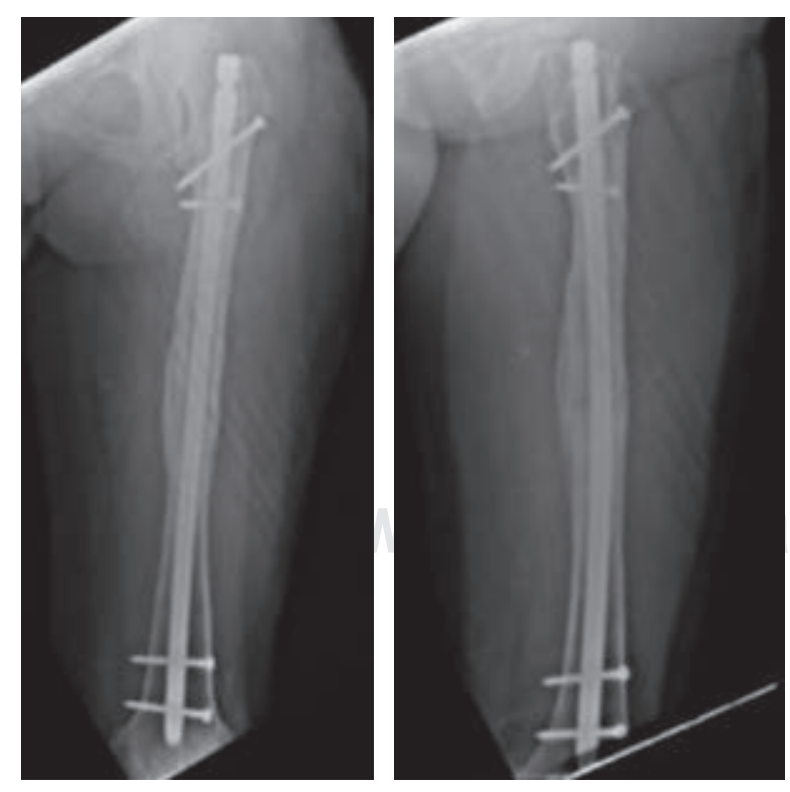

Figura 5: Seguimiento a cuatro años. sus siglas en inglés). Un estudio investigó la eficacia del PRP en el tratamiento de 132 pacientes con retardo en la consolidación en fracturas de huesos largos tratados entre 2009 y 2012, la consolidación fue establecida en 108 pacientes (81.8\%) después de administrar PRP. En otro estudio con 94 pacientes se demostró la eficacia del PRP en el tratamiento de no unión de huesos largos encontrando que 82 pacientes (87.23\%) consolidaron a los cuatro meses y no presentaron complicaciones. ${ }^{17}$ Por lo anterior, utilizamos el PRP como una piedra angular en nuestra técnica.

El uso de aloinjertos se considera como parte estructural del defecto; sin embargo, ha mostrado resultados pocos favorables en su integración. ${ }^{18-20}$ Contrario a lo observado en este caso.

La aplicación del «concepto diamante» que consiste en el uso de aspirado de médula, PRP y matriz ósea desmineralizada provee las condiciones óptimas mecánico-biológicas para la reparación ósea. ${ }^{21}$ Por lo anterior, se consideró agregar PRP como coadyuvante en nuestro procedimiento.

Issaoui y colaboradores reportaron el caso de una paciente de 16 años de edad, con fractura expuesta de fémur grado III-A con pérdida ósea de $8 \mathrm{~cm}$, a quien se realizó la técnica de inducción de membrana; en la primera fase, colocación de placa de compresión dinámica más polimetilmetacrilato en el área del defecto y 11 semanas después, retiro del PMMA-cemento más colocación de aloinjerto en chips de esponjosa, logrando una consolidación completa a los 15 meses; sin embargo, fue capaz de reincorporarse a sus actividades hasta los 20 meses. A los ocho meses de lograr la consolidación completa, le retiran la placa y sufre una refractura en el mismo sitio, por lo cual fue necesaria una reintervención, la colocación de clavo centromedular de fémur anterógrado más autoinjerto, iniciando la descarga de peso a los tres meses, logrando una mejoría tanto clínica como radiográfica hasta ocho meses después de la reintervención. ${ }^{22}$ En comparación con nuestros resultados, el apoyo se inició al finalizar el primer mes de postoperada y en un solo tiempo quirúrgico.

En un artículo publicado por Vejarano y colaboradores se describe un caso de paciente femenino de 20 años con diagnóstico de pseudoartrosis atrófica de fémur, tratada inicialmente con clavo de centromedular bloqueado, con colocación de placa a los 12 meses para mejorar la estabilidad, con persistencia de pseudoartrosis; se coloca injerto óseo autólogo y plasma rico en plaquetas, a las 20 semanas posteriores se muestra consolidación completa en control radiográfico, ${ }^{23}$ lo cual coincide con nuestros resultados. 
Neogi y colaboradores presentan un caso de pérdida ósea en fémur por hidatidosis ósea en paciente femenino de 35 años, a quien en un primer tiempo se le colocó una placa de compresión de bloqueo (LCP) 4.5 con polimetilmetacrilato (PMMA) en la pérdida ósea y posteriormente se retira el PMMA y ante una pérdida de $24 \mathrm{~cm}$ se coloca aloinjerto diafisario con clavo centromedular, consolidando de manera completa a los 24 meses. ${ }^{24} \mathrm{~A}$ diferencia del nuestro, que se integró completamente a los 20 meses y en una sola intervención.

En nuestro caso clínico tuvimos la limitación en cuanto a la falta de aplicación de pruebas funcionales validadas para realizar el análisis más completo de resultados del tratamiento aplicado en nuestra paciente.

\section{Conclusiones}

En nuestro caso podemos observar que, adicionando plasma rico en plaquetas en la utilización de aloinjerto de segmento diafisario de fémur y con una adecuada estabilidad, la paciente logró una completa consolidación ósea y funcionalidad, recuperando la movilidad y la longitud de su extremidad afectada, además de una deambulación independiente y una integración a sus actividades habituales, logrando una excelente calidad de vida. Actualmente, a cuatro años se encuentra desde el punto de vista radiográfico con una total consolidación e integración del injerto y clínicamente con una funcionalidad normal.

Se abre un nuevo panorama a la ortobiología, ya que forma un rol importante en la consolidación ósea; cada día con mayor soporte científico de que las terapias biológicas tienen un futuro prometedor a corto plazo, sopesando el riesgo costo-beneficio. Las pérdidas óseas masivas son un desafío para el ortopedista, por lo cual hoy en día debe estar dentro de nuestro arsenal terapéutico el uso de ortobiológicos y dentro de éstos, el uso del plasma rico en plaquetas.

\section{Referencias}

1. Schmal H, Brix M, Bue M, Ekman A, Ferreira N, Gottlieb H, et al. Nonunion - consensus from the 4th annual meeting of the Danish Orthopaedic Trauma Society. EFORT Open Rev. 2020; 5 (1): 46-57.

2. Nandra R, Grover L, Porter K. Fracture non-union epidemiology and treatment. Trauma. 2016; 18 (1): 3-11.

3. Sen C, Eralp L, Gunes T, Erdem M, Ozden VE, Kocaoglu M. An alternative method for the treatment of nonunion of the tibia with bone loss. J Bone Joint Surg Br. 2006; 88 (6): 783-789.

4. Pihlajamaki HK, Salminen ST, Bostman OM. The treatment of nonunions following intramedullary nailing of femoral shaft fractures. J Orthop Trauma. 2002; 16 (6): 394-402.
5. Stewart SK. Fracture non-union: a review of clinical challenges and future research needs. Malays Orthop J. 2019; 13 (2): 1-10.

6. Adamczyk A, Meulenkamp B, Wilken G, Papp S. Managing bone loss in open fractures. OTA Int. 2020; 3 (1): e059.

7. Haines NM, Lack WD, Seymour RB, Bosse MJ. Defining the lower limit of a "critical bone defect" in open diaphyseal tibial fractures. J Orthop Trauma. 2016; 30 (5): 158-163.

8. Keating JF, Simpson AH, Robinson CM. The management of fractures with bone loss. J Bone Joint Surg Br. 2005; 87 (2): 142-150.

9. Cavanna M. WITHDRAWN: Allograft for the treatment of massive bone loss in open and infected IIIA fracture of the distal femur: a case report. Injury. 2021. Available in: https:// doi.org/10.1016/j.injury.2018.11.046

10. Mendieta A, Soriano JC, Negrete CJ. Utility of platelet-rich plasma and growth factors bone in the bone defects. Acta Ortop Mex. 2007; 21 (5): 256-260.

11. Romo RJ, Gámez SL, Escriva MJ. Factores de crecimiento en cirugía ortopédica. Orthotips. 2009; 5 (1): 88-92.

12. Fernández-Fairen $M$, Gutiérrez-Gómez J, Hernández-Vaquero D, Torres-Pérez Al, Sueiro-Fernández J. Evidencia científica de los factores de crecimiento en cirugía ortopédica y traumatología. Orthotips. 2014; 10 (4): 235-257.

13. Calori GM, Colombo M, Mazza EL, Mazzola S, Malagoli E, Marelli N, et al. Validation of the Non-Union Scoring System in 300 long bone non-unions. Injury. 2014; 45 Suppl 6: S93-S97.

14. Alford Al, Nicolaou D, Hake M, McBride-Gagyi S. Masquelet's induced membrane technique: Review of current concepts and future directions. J Orthop Res. 2021; 39 (4): 707-718.

15. Masquelet A, Kanakaris NK, Obert L, Stafford P, Giannoudis PV. Bone repair using the Masquelet technique. J Bone Joint Surg Am. 2019; 101 (11): 1024-1036.

16. Dallo I, Etcheto HFR, Collazo C, Chahla J, Gobbi A. Terapias biológicas en artroscopia de rodilla. De las ciencias básicas a la aplicación clínica. Artroscopia. 2020; 27: 136-145.

17. Wang W, Yeung KWK. Bone grafts and biomaterials substitutes for bone defect repair: a review. Bioact Mater. 2017; 2 (4): 224-247.

18. Vastel L. Aloinjertos óseos y bancos de tejidos. EMC - Apar Locomot. 2010; 43 (3): 1-10.

19. Mainard D. Sustitutos óseos. EMC - Apar Locomot. 2014; 47 (2): 1-11.

20. Balakrishnan M, Agarwal DC, Kumar S. A study of efficacy of heterogeneous bone grafts (surgibone) in orthopaedic surgery. Med J Armed Forces India. 2000; 56 (1): 21-23.

21. Rodriguez-Collazo E, Khan AA, Khan HA. An algorithmic approach towards the orthoplastic management of osseous and soft tissue defects in post-traumatic distal tibial fractures. J Orthop Trauma Surg Rel Res. 2017; 12 (2): 56-61.

22. Issaoui H, Fekhaoui MR, Jamous M, Masquelet AC. Modified masquelet technique using allogeneic graft for a GustiloAnderson type III-A open fracture of the femur with an $8 \mathrm{~cm}$ bone defect. Case Rep Orthop. 2021; 2021: 8829158.

23. Vejarano-Solano JC, Shu-Yip SB, Ruiz-Semba CF, VidalEscudero JA. Uso de plasma rico en plaquetas autólogo en el tratamiento quirúrgico de pseudoartrosis atrófica de fémur y tibia. Rev Med Hered. 2013; 24 (2): 122-130.

24. Neogi DS, Kumar V, Malhotra R. Femoral allograft in the management of osseous hydatidosis presenting as femoral shaft non-union. Arch Orthop Trauma Surg. 2009; 129 (12): 1627-1632.

\section{Conflicto de intereses}

No hay conflicto de intereses. 